\title{
Laudatio: Verleihung des DVPW-Förderpreises für Dissertationen an Ulrike Klinger
}

\author{
Rainer Eising / Doris Fuchs / Matthias Bohlender
}

2012 waren 15 Arbeiten für den DVPW-Dissertationspreis nominiert. Mit Hilfe der von einschlägigen Expertinnen und Experten eingeholten Fachgutachten haben sich die Mitglieder der Jury dafür entschieden, diesen Preis an Ulrike Klinger für ihre Studie „Medienmonopole in defekten Demokratien. Media Governance und die Aushandlung von Pluralismus in Italien und Mexiko" zu verleihen. Die Arbeit wurde 2009 an der Goethe-Universität Frankfurt eingereicht und aufgrund der dortigen Gutachten von Prof. Dr. Hans-Jürgen Puhle und Prof. Dr. Marianne Braig als Promotionsschrift angenommen. Sie ist 2011 als Band 13 in der renommierten Reihe „Politische Kommunikation und demokratische Öffentlichkeit“ (Nomos Verlag, Baden-Baden) publiziert worden.

Die Studie verknüpft die Medien- und Kommunikationsforschung mit Einsichten der Demokratisierungs- und Transformationsforschung. Medien und Medienberichterstattung sind für Frau Klinger lohnenswerte Untersuchungsgegenstände, weil sie einerseits wirtschaftliche Güter und andererseits regulierungsbedürftige öffentliche Güter darstellen, die die politische Interessenvermittlung in modernen Massendemokratien erst möglich machen. Medienregulierung müsse demnach sowohl politische Öffentlichkeit befördern als auch die wirtschaftlichen Bedürfnisse von Medienunternehmen berücksichtigen. Im Mittelpunkt der Analyse steht die Bedeutung von Medienkonzentration für die Demokratie in zwei defekten Demokratien. Aus der Medien- und Kommunikationsforschung leitet die Autorin ab, dass Medienoligopole Medienpolitik bestimmen, Wettbewerb blockieren und Wahlkämpfe manipulieren könnten. Dem hält sie entgegen, dass Demokratien „dem Prozess der Medienkonzentration und deren potenziellen Folgen nicht hilflos gegenüber stehen " (S. 13), sondern diesen durchaus steuern können. Diese Thesen analysiert sie mit Hilfe eines systematischen und mehrschichtigen qualitativen Forschungsdesigns.

Als Untersuchungsländer wählt Frau Klinger Mexiko und Italien. Beide Länder weisen im internationalen Vergleich eine besonders starke Medienkonzentration im Rundfunk- und Fernsehsektor auf, sodass sich hier für die Demokratie besonders abträgliche Folgen identifizieren lassen müssten. Gleichzeitig variieren die beiden Länder in ihren Systemeigenschaften und im Grad ihrer demokratischen Reife. Mexiko sei eine junge, noch nicht konsolidierte Demokratie mit einem konkurrenzorientierten politischen System, das auf den Präsidenten ausgerichtet sei. Dagegen wird Italien als eine dekonsolidierte Demokratie mit größerer Reife präsentiert, die ein eher konsensorientiertes parlamentarisches System aufweise. Frau Klinger untersucht also die Bedeutung der Medienkonzentration für die Demokratie in verschiedenen nationalen Kontexten, um so jene Faktoren zu identifizieren, die sich auf die Regulierung von Medienpluralismus auswirken. Dies erfolgt unter Nutzung der Vetospielertheorie mittels eingebetteter vergleichender 
Fallstudien innerhalb des Zeitraums von 2000 bis 2007: Frau Klinger vergleicht je zwei medienpolitische Gesetzgebungsprozesse in beiden Ländern miteinander, untersucht die Aktivitäten der Medienoligopole zur Verhinderung eines stärkeren Medienpluralismus und analysiert die Wirkung der Medienkonzentration auf die Berichterstattung zu Wahlkämpfen in beiden Ländern. Die empirische Analyse stützt sich auf qualitative Dokumentenanalysen, Experteninterviews und die Analyse von Primärdaten zum Medienmonitoring der Wahlkämpfe, soweit verfügbar. Die Autorin demonstriert hier eine große Souveränität im Umgang mit qualitativen und quantitativen Daten.

Die ausgezeichnet informierte Untersuchung weist u. a. nach, dass die langfristigen Tendenzen zur Medienkonzentration in beiden Ländern durchaus politisch gewollt waren. Beide Länder förderten lange Zeit die Abhängigkeit der öffentlichen Medien (Italien) vom Staat oder auch die enge Kopplung der privaten Medien an Parteien und Staat (Mexiko). Die zentralen Medienunternehmen in beiden Ländern waren im Untersuchungszeitraum in der Lage, maßgeblichen Einfluss auf die staatliche Medienpolitik zu nehmen. In Italien haben die medienpolitischen Interessen des Ministerpräsidenten Berlusconi einer unternehmerfreundlichen Rundfunkreform den Weg bereitet, während in Mexiko die Medienunternehmen eine Reform blockieren konnten, die auf eine pluralistischere Struktur der Rundfunkmedien abzielte, weil Parteipolitiker hier eine hohe Abhängigkeit von der Medienberichterstattung in nationalen Wahlkämpfen wahrgenommen haben. Frau Klinger weist allerdings auch nach, dass diese Reformen nicht unumstritten waren, sondern starke Gegenbewegungen nach sich gezogen haben: In Italien sei eine von der kurzlebigen Prodi-Regierung 2006-08 angestrebte Reform der Reform aufgrund unzureichender Mehrheiten in den beiden Kammern gescheitert. In Mexiko wurde dagegen eine Verfassungsreform beschlossen, die die Medienmacht in der Wahlberichterstattung deutlich reduziert hat. Als zentrale Akteure für die zukünftige Regulierung des Mediensektors und die Erhöhung des Pluralismus in den Medien als ein Element in der (Re-)Konsolidierung der Demokratie werden die politischen Parteien in Italien und Mexiko ausgemacht. Das Beispiel Italien belege überdies, dass eine stärkere Trennung privater Medieninteressen von staatlichen Ämtern und Aufgaben vonnöten sei.

Frau Klinger verknüpft in ihrer Studie nicht nur auf innovative Art und Weise verschiedene politikwissenschaftliche Themenbereiche und untersucht diese systematisch in einer theoriegeleiteten empirischen Analyse. Die Autorin reflektiert auch die Reichweite ihrer Erklärungen, sucht die genutzten Theorien zu modifizieren, diskutiert die grundlegende Bedeutung ihrer Befunde für die Demokratieforschung und entwickelt normative Schlussfolgerungen für die Regulierung von Mediensystemen. Die Jurymitglieder gratulieren der Preisträgerin herzlich und wünschen ihr für die Zukunft weiterhin viel Erfolg. Wir schulden zudem jenen Kolleginnen und Kollegen großen Dank, deren Fachgutachten unsere Auswahl vorbereitet haben. 